\title{
A case-control study of occupation/industry and renal cell carcinoma risk
}

\author{
Sara Karami ${ }^{*}$, Joanne S Colt ${ }^{1}$, Kendra Schwartz ${ }^{2}$, Faith G Davis ${ }^{3}$, Julie J Ruterbusch², \\ Stella S Munuo ${ }^{4}$, Sholom Wacholder ${ }^{1}$, Patricia A Stewart ${ }^{5,6}$, Barry I Graubard ${ }^{1}$, \\ Nathanial Rothman ${ }^{1}$, Wong-Ho Chow ${ }^{1}$ and Mark P Purdue ${ }^{1}$
}

\begin{abstract}
Background: The role of occupation in the etiology of renal cell carcinoma (RCC) is unclear. Here, we investigated associations between employment in specific occupations and industries and RCC, and its most common histologic subtype, clear cell RCC (ccRCC).

Methods: Between 2002 and 2007, a population-based case-control study of Caucasians and African Americans $(1,217$ cases; 1,235 controls) was conducted within the Detroit and Chicago metropolitan areas to investigate risk factors for RCC. As part of this study, occupational histories were ascertained through in-person interviews. We computed odds ratios (ORs) and 95\% confidence intervals (Cls) relating occupation and industry to RCC risk using adjusted unconditional logistic regression models.

Results: Employment in the agricultural crop production industry for five years or more was associated with RCC $(\mathrm{OR}=3.3[95 \% \mathrm{Cl}=1.0-11.5])$ and $\mathrm{ccRCC}$ in particular $(\mathrm{OR}=6.3[95 \% \mathrm{Cl}=1.7-23.3], P$ for trend with duration of employment $=0.0050$ ). Similarly, RCC risk was elevated for employment of five years or longer in non-managerial agricultural and related occupations $\left(\mathrm{OR}_{\mathrm{RCC}}=2.1[95 \% \mathrm{Cl}=1.0-4.5] ; \mathrm{OR}_{\mathrm{CCRCC}}=3.1[95 \% \mathrm{Cl}=1.4-6.8]\right)$. Employment in the dry-cleaning industry was also associated with elevated risk $\left(\mathrm{OR}_{\mathrm{RCC}}=2.0[95 \% \mathrm{Cl}=0.9-4.4], P\right.$ for trend $=0.093$; $\mathrm{OR}_{\mathrm{CCRCC}}=3.0[95 \% \mathrm{Cl}=1.2-7.4]$, $P$ for trend $=0.031$ ). Suggestive elevated associations were observed for police/ public safety workers, health care workers and technicians, and employment in the electronics, auto repair, and cleaning/janitorial services industries; protective associations were suggested for many white-collar jobs including computer science and administrative occupations as well employment in the business, legislative, and education industries.
\end{abstract}

Conclusions: Our findings provide support for an elevated risk of RCC in the agricultural and dry-cleaning industries and suggest that these associations may be stronger for the ccRCC subtype. Additional studies are needed to confirm these findings.

Keywords: Kidney cancer, Renal cancer, Clear cell RCC, Occupation, Industry, Race

\section{Background}

Malignant tumors of the kidney account for about 2\% of cancer diagnoses worldwide [1]. In the United States (U.S.), kidney cancer accounts for approximately $4 \%$ of newly diagnosed cancer cases and $2 \%$ of cancer deaths $[2,3]$. The most common form, renal cell carcinoma

\footnotetext{
* Correspondence: karamis@mail.nih.gov

'Division of Cancer Epidemiology and Genetics, National Cancer Institute, NIH, DHHS, 6120 Executive Boulevard, MSC 7242, Bethesda, MD 20892-7242, USA

Full list of author information is available at the end of the article
}

(RCC) of the renal parenchyma, accounts for more than $85 \%$ of kidney cancers $[3,4]$. RCC includes several histologic subtypes, the most common of which is clear cell RCC (ccRCC), making up approximately $70 \%$ of cases [5]. These subtypes possess different genetic, clinical, and demographic characteristics [5,6]; differences in etiology have also been speculated [7].

The etiology of RCC is complex and not well understood. Cigarette smoking, excess body weight and hypertension are well established risk factors that account for nearly half of all RCC diagnoses in the U.S. [1,3,4].

\section{Biomed Central}


Although generally not considered an occupational disease, an association between RCC and occupational risk factors has been suggested in a number of epidemiological studies [4,8-16]. Most recently, a large Eastern European case-control study reported statistically significant increased RCC risk for workers in the agricultural and animal husbandry industries, particularly among farmers [8]. Other industries and occupations that have been linked to RCC risk, although not consistently, include printers [3,9-11], mechanics and repairers $[3,9,12]$, metal workers $[3,8-11,13]$, truck drivers $[13,14]$, railroad workers $[3,10,11,13]$, aircraft mechanics [3,9], and those employed in the dry-cleaning [9,15], petroleum $[10,13,15]$, iron and steel $[4,13,16]$, and printing $[9,10,13]$ industries. To our knowledge, no studies of occupation and RCC have investigated associations with RCC subtypes.

To further explore the relationship between occupation and RCC risk, we analyzed lifetime occupational histories collected from participants of a populationbased case-control study of Caucasians and African Americans conducted in the U.S. The study was designed to explore a variety of risk factors in the etiology of RCC, and to examine whether the risk factors varied by race.

\section{Methods}

\section{Study population}

Caucasian and African American male and female residents of Chicago, Illinois (Cook County) and Detroit, Michigan (Macomb, Oakland, and Wayne Counties) were the source population for this study. All incident cases of histologically confirmed adenocarcinoma of the kidney (ICD-O C64) between 20 and 79 years of age diagnosed within the enrollment periods for Chicago (January 1, 2003 through December 31, 2003) and Detroit (February 1, 2002 through January 31, 2007 for African Americans and through July 31, 2006 for Caucasians) were eligible to participate. Potential cases from the Detroit area were identified through the Metropolitan Detroit Cancer Surveillance System, a cancer registry of the National Cancer Institute's Surveillance, Epidemiology, and End Results (SEER) program. In Chicago, potential cases were identified from pathology reports issued at participating hospitals in Cook County and adjacent communities. Controls were recruited from the general population, with frequency matching to the case series on the basis of age group, self-reported race, sex, and study center. Controls aged 65-79 years were identified from files of the Centers for Medicare and Medicaid Services, and controls under age 65 years were identified from Department of Motor Vehicle (DMV) records.

Although RCC rates are higher among African Americans than Caucasians [3], many more Caucasian than
African American RCC cases were diagnosed in the study areas, as expected given the greater number of Caucasian residents. This limited power for analysis of risk factors by race. Therefore, a sampling strategy was designed to recruit a sufficient number of African Americans efficiently [17], that is, without exceeding recruitment goals for Caucasians. All African American cases were recruited, while some age-sex strata of Caucasian cases were subsampled. To further increase power for analyses restricted to African Americans, the study maintained a control:case matching ratio of 2:1 for African Americans. For Caucasians, with larger numbers of cases, there was less need for additional power, and we therefore matched at a ratio of 1:1. Information on race was unavailable from DMV records, hampering our ability to frequency match controls to the cases among those 20 to 64 years of age. Therefore, we used the racial density of the census block group (according to the U.S. 2000 Census) in which each control resided as a surrogate for race for the purposes of sampling, and over-sampled people living in high-density African American areas [17].

Of the 1,918 eligible cases identified, 171 died prior to contact or interview, 92 could not be located with the available contact information, 21 moved out of the area, and the physicians of 63 cases refused permission to contact their patients. Among the remaining 1,571 cases we sought to enroll, 221 declined participation and 133 were not interviewed due to serious illness, impairment, or not responding to multiple attempts to contact. Thus, 1,217 cases (77.5\% of those we attempted to recruit) participated in the study. Of the 2,718 presumed eligible controls, 41 died prior to contact or interview, 345 could not be located with the available contact information, and 63 had moved out of the region. Among the 2,269 controls we attempted to recruit, 677 declined to participate and 357 were not interviewed due to serious illness, impairment, or not responding to multiple attempts to contact. Thus, 1,235 eligible controls $(54.4 \%$ of those we attempted to recruit) participated. Approvals were obtained from human subjects review boards at all participating institutions, and informed written consent was obtained from all participants.

Copies of medical records were obtained from all cases to confirm diagnosis and collect information on histologic and clinical factors. In addition, the original diagnostic slides were obtained for 706 cases for review by an experienced pathologist. We assigned histology on the basis of the centralized histopathologic review if available; otherwise, information from the original diagnostic pathology reports was used.

\section{Data collection}

Those who agreed to participate were scheduled for an in-home, computer-assisted personal interview. Prior to 
the interview, a work history calendar was mailed to the home, and participants were asked to record information on job title, tasks performed, equipment and chemicals regularly used, and years employment began and ended, for all jobs that were held for at least 12 months. Trained interviewers reviewed the work history calendars at the time of the interview to ensure that the data was complete as they entered the information into the computer. Other information collected during the inhome interview included data on demographics, smoking history, medical and medication history, diet, and family history of cancer.

\section{Occupational coding and statistical analysis}

The Standard Occupational Classification (SOC) [18] and Standard Industry Classification (SIC) [19] schemes were used to code each job held by each participant. RCC and ccRCC risk was estimated for ever/never employment and by duration of employment (never [referent], $<5$ years, $\geq 5$ years), for every two-, three-, and four-digit SIC and SOC code. Results are presented in the tables only for occupations and industries held by at least 10 study participants. Additional file 1: Table S1 and Additional file 2: Table S2 provide results for all subjects combined, and separately by sex and race, for every industry and occupation reported.

Results are presented first for occupations and industries suspected a priori to be associated with RCC risk. A priori jobs were determined by reviewing the literature and identifying all occupations or industries significantly $(P$-value $\leq 0.05)$ associated with kidney cancer risk in at least two published studies. All studies written in English, and identified in PubMed using the keywords kidney cancer and occupation, kidney cancer and industry, or kidney cancer and jobs, were examined. Results for $a$ posteriori high- and low-risk occupations and industries as well as for ccRCC are presented only if we observed (1) a significant association with ever employment or (2) both a significant association with duration of employment and, to increase the likelihood of capturing duration-response relationships that were monotonic in nature, a $P$-value for ever employment of 0.10 or lower.

For analytic purposes, a set of sample weights was developed to reduce the potential for bias arising from differential sampling rates for controls and cases, from survey nonresponse, and from deficiencies in the coverage of the population at risk by the files of the DMV and Centers for Medicare and Medicaid Services to select the controls. Sample weights also include a poststratification adjustment so that the weighted distribution of controls across the matching variables matched exactly the weighted distribution of cases. In addition to being consistent with the objectives of the frequency matching, this poststratification adjustment reduces the variability of the weights [20]. Full detail of the development of the sample weights has been described previously [17].

The sample-weighted frequency distributions of selected characteristics and known RCC risk factors were compared between cases and controls using a Wald F-test [21]. Unconditional logistic regression models using poststratified weights were used to calculate ORs and 95\% CIs associated with work history and duration of employment, using individuals never employed in the occupation or industry as the reference group. Tests for trend were performed by modeling medians of employment duration as an ordinal variable and applying the Wald Chi-Square test [21]. The jackknife replicate weight method was used to estimate standard errors [22]. Regression models were adjusted for RCC risk factors which included self-reported hypertension history (ever, never), smoking status (never, occasional, former, current), BMI (self-reported height and weight five years prior to interview) as well as sex, age (at diagnosis for cases and at study selection for controls), race, and family history of cancer. Regression models were additionally adjusted for study center and level of education given that an individual's work environment and potential occupational exposures is related to these factors. Because previously published studies have shown a link between hypertension and certain occupational exposures (i.e., lead and cadmium) [23,24], analyses were also assessed excluding hypertension from the model; however, no new significant associations were observed. Unweighted unconditional logistic regression analyses for ever employment and duration of employment were also conducted; results were similar to those of the weighted analyses [results not shown]. All analyses were conducted with SAS version 9.2 [SAS Institute, Cary, $\mathrm{NC}$, USA] using procedures appropriate for sample weighted data. Statistical tests were determined to be significant at a two-sided $P$-value $<0.05$.

\section{Results}

Cases and controls were comparable in sex and age distributions (Table 1). Cases were more likely than controls to have a lower education level $(P<0.001)$, be current smokers $(P=0.03)$, have a history of hypertension $(P<0.001)$, and have excess body weight (body mass index $\left.(\mathrm{BMI})>30 \mathrm{~kg} / \mathrm{m}^{2}\right)(P<0.001)$.

RCC risk associations for all occupations and industries chosen a priori are shown in Table 2. There were no occupations or industries for which RCC risk increased significantly as duration of employment increased, although a trend of borderline significance was observed for the agricultural crop production industry (Standard Industry Classification (SIC) 01: $P$ for trend $=0.051$ ), with a three-fold elevated risk among 
Table 1 Characteristics of USRCC Cases and Controls

\begin{tabular}{llllll}
\hline & \multicolumn{4}{c}{ Weighted distribution } \\
\cline { 2 - 3 } Variables & \multicolumn{3}{c}{ Cases } & & \multicolumn{2}{c}{ Controls } \\
\cline { 2 - 3 } & $\mathbf{N}$ & $\mathbf{\%}^{\mathbf{a}, \mathbf{b}}$ & & $\mathbf{N}$ & $\mathbf{\%}^{\mathbf{a}, \mathbf{b}}$ \\
\hline Total & 1,217 & & & 1,235 & \\
\hline Race & 856 & 73.9 & & 712 & 73.9 \\
\hline Caucasian & 361 & 26.1 & & 523 & 26.1 \\
\hline African American & & & &
\end{tabular}

\begin{tabular}{lllll}
\hline Sex & & & & \\
\hline Males & 720 & 61.8 & 689 & 61.4 \\
\hline Females & 497 & 38.2 & 546 & 38.6 \\
\hline
\end{tabular}

\begin{tabular}{lllll}
\hline Age at Reference Date & & & & \\
\hline$<45$ & 147 & 10.5 & 179 & 10.5 \\
\hline $45-54$ & 287 & 21.6 & 270 & 21.6 \\
\hline $55-64$ & 372 & 29.4 & 350 & 29.4 \\
\hline $65-74$ & 303 & 27.1 & 329 & 27.1 \\
\hline $75+$ & 108 & 11.5 & 107 & 11.5 \\
\hline
\end{tabular}

\begin{tabular}{lllll}
\hline Mean Age & 59.9 years & \multicolumn{2}{l}{59.9 years } \\
\hline Study Center & \multicolumn{3}{l}{} \\
\hline Detroit & 1,018 & 83.3 & 1,038 & 82.7 \\
\hline Chicago & 199 & 16.7 & 197 & 17.3 \\
\hline
\end{tabular}

\section{Education Level}

\begin{tabular}{lllll}
\hline$<12$ years & 200 & 16.7 & 165 & 12.0 \\
\hline High School Graduate & 419 & 34.5 & 390 & 31.5 \\
\hline Some College & 328 & 26.3 & 356 & 27.3 \\
\hline College Graduate & 270 & 22.5 & 324 & 29.3 \\
\hline
\end{tabular}

\section{Smoking Status}

\begin{tabular}{lllll}
\hline Never & 432 & 35.3 & 471 & 38.4 \\
\hline Occasional $^{c}$ & 55 & 4.7 & 55 & 4.0 \\
\hline Regular Former Smoker & 410 & 34.7 & 445 & 38.0 \\
\hline Regular Current Smoker & 320 & 25.3 & 264 & 19.7 \\
\hline
\end{tabular}

\section{History of Hypertension}

\begin{tabular}{lllll}
\hline No & 500 & 40.8 & 718 & 59.0 \\
\hline Yes & 701 & 59.2 & 508 & 41.0 \\
\hline BMI $\left(\mathbf{k g} / \mathbf{m}^{\mathbf{2}}\right)^{\text {d }}$ & & & & \\
\hline$<25$ & 240 & 19.5 & 366 & 29.1 \\
\hline $25-29.9$ & 436 & 37.4 & 493 & 41.7 \\
\hline $30-34.99$ & 298 & 24.9 & 221 & 18.3 \\
\hline $35+$ & 230 & 18.2 & 147 & 10.9 \\
\hline Histologic RCC Subtype & & & & \\
\hline Clear Cell & 709 & 58.3 & & \\
\hline Papillary & 169 & 13.9 & & \\
\hline Chromophobe & 58 & 4.8 & & \\
\hline Other/NOS & 281 & 23.1 & & \\
\hline a Due to rounding eror & & & \\
\hline
\end{tabular}

${ }^{a}$ Due to rounding error, some categories do not sum to $100 \%$. ${ }^{\mathrm{b}} \mathrm{A}$ sample weighted frequency distribution. ${ }^{\mathrm{C}}$ Smoked 100 cigarettes in the lifetime, but never smoked $>1$ cigarette a day for $>6$ months. ${ }^{d}$ BMI five years prior to interview. The following data are unknown: BMI (13 cases, 8 controls), history of hypertension (16 cases, 9 controls). individuals employed for five or more years (odds ratio $(\mathrm{OR})=3.3[95 \%$ confidence interval $(\mathrm{CI})=1.0-11.5])$. Patterns in this industry were similar for men and women (Additional file 1: Table S1). Significantly increased RCC risk for employment of five years or longer was also observed for agricultural and related occupations, excluding farm managers and proprietors (Standard Occupational Classification (SOC) 56: OR $=2.1 \quad[95 \%$ $\mathrm{CI}=1.0-4.5], P$ for trend $=0.094$ ), and in particular for non-managerial farm occupations (SOC 561: $\mathrm{OR}=3.2$ $[95 \% \mathrm{CI}=1.0-10.1], P$ for trend $=0.060)$. There was a suggestion of elevated risk in the dry-cleaning plant industry (SIC 7216: OR for ever employed $=2.0[95 \%$ $\mathrm{CI}=0.9-4.4]$, increasing to $\mathrm{OR}=2.5[95 \% \mathrm{CI}=0.4-14.4]$ for employment of $\geq 5$ years $(P$ for trend $=0.093)$ ), particularly among men (Additional file 1: Table S1). We observed a significant reduction in risk with increasing duration of employment as a mechanic or repairer (SOC 61: $P$ for trend $=0.038$ ). Stratified analyses by race or sex showed no noteworthy differences in association for other a priori jobs (Additional file 1: Table S1 and Additional file 2: Table S2).

RCC risks for a posteriori high- and low-risk occupations are shown in Table 3. Significant elevations in RCC risk, with significant trends with employment duration, were observed for health technologists and technicians (SOC 36: $\mathrm{OR}=1.7 \quad[95 \% \mathrm{CI}=1.1-2.6], P$ for trend $=$ 0.043), pressing machine operators (SOC 7657: $\mathrm{OR}=4.7$ [95\% CI $=1.3-17.4], P$ for trend $=0.044)$, and machine feeders and off bearers (SOC 8725: OR $=2.2[95 \% \mathrm{CI}=$ 1.1-4.3], $P$ for trend $=0.024$ ). A nearly five-fold risk was observed among those ever employed as recreational workers (SOC 2033: OR $=4.8$ [95\% CI = 1.7-13.9]). Stratification by sex and race (Additional file 2: Table S2) revealed a small number of additional occupations with significant associations for ever employment and significant trends with duration of employment: male insurance workers (SOC 4122: $\mathrm{OR}=2.2[95 \% \mathrm{CI}=1.1-4.4], P$ for trend $=0.026$ ), female janitors and cleaners (SOC 5244: $\mathrm{OR}=2.8[95 \% \mathrm{CI}=1.2-6.7], P$ for trend $=0.044)$, and Caucasian sales workers (SOC 42: OR $=1.6[95 \%$ $\mathrm{CI}=1.1-2.5], \quad P$ for trend $=0.022$ ). Several statistically significant protective associations were also observed in Table 3, mainly for white- collar occupations such as administrators, computer scientists and programmers, librarians, and various administrative support occupations.

Table 4 shows RCC risk associations for a posteriori high- and low-risk industries. RCC risk was significantly elevated with ever and duration of employment for security/commodity brokers and services (SIC 62: OR $=3.2$ [95\% CI $=1.2-8.7], P$ for trend $=0.015$ ) and for police protection (SIC 9221: OR $=2.2[95 \% \mathrm{CI}=1.0-4.8], P$ for trend $=0.045$ ). Increased RCC association for employment of five years or longer was seen for those in the 
Table 2 Risk of renal cell carcinoma for a priori occupations and industries

\begin{tabular}{|c|c|c|c|c|c|c|c|c|c|}
\hline \multirow[t]{2}{*}{ Job Code \& Description } & \multirow{2}{*}{\multicolumn{2}{|c|}{$\frac{\text { NEVER }}{\text { Case/Control }}$}} & \multicolumn{2}{|c|}{ EVER } & \multicolumn{2}{|c|}{$<5$ YEARS } & \multicolumn{3}{|c|}{$5+$ YEARS } \\
\hline & & & Case/Control & $\mathrm{OR}^{\mathrm{a}}[95 \% \mathrm{Cl}]$ & Case/Control & $\mathrm{OR}^{\mathrm{a}}[95 \% \mathrm{Cl}]$ & Case/Control & $\mathrm{OR}^{\mathrm{a}}[95 \% \mathrm{Cl}]$ & $P$-trend \\
\hline \multicolumn{10}{|l|}{ OCCUPATION: } \\
\hline SOC 11-13: officials and administrators, other & $983 / 1003$ & 1.0 & $201 / 208$ & $0.9[0.7-1.2]$ & $38 / 57$ & $0.7[0.4-1.1]$ & $163 / 151$ & $1.0[0.8-1.4]$ & 0.81 \\
\hline SOC 1633: electrical and electronic engineers & $1177 / 1199$ & 1.0 & $7 / 12$ & $0.6[0.2-2.0]$ & $1 / 3$ & $0.2[0.0-1.6 \mathrm{E}+09]$ & $6 / 9$ & $0.8[0.2-3.1]$ & 0.67 \\
\hline SOC 42: sales occupations, commodities except retail & $1122 / 1161$ & 1.0 & $62 / 50$ & $1.4[0.9-2.1]$ & $14 / 10$ & $1.4[0.6-3.1]$ & $48 / 40$ & $1.4[0.9-2.1]$ & 0.088 \\
\hline SOC 43: sales occupations, retail & 903/927 & 1.0 & $281 / 284$ & $1.0[0.9-1.2]$ & $186 / 179$ & $1.1[0.9-1.4]$ & $95 / 105$ & $0.9[0.7-1.3]$ & 0.76 \\
\hline SOC 5123: firefighting occupations & $1176 / 1204$ & 1.0 & $8 / 7$ & $1.4[0.4-4.7]$ & $3 / 1$ & $3.2[0.0-8.8 \mathrm{E}+09]$ & $5 / 6$ & $1.1[0.3-4.8]$ & 0.81 \\
\hline SOC 551: farmers (working proprietors) & $1178 / 1207$ & 1.0 & $6 / 4$ & $2.4[0.5-11.3]$ & $5 / 3$ & $2.4[0.4-15.5]$ & $1 / 1$ & $2.2[0.0-5.3 E+13]$ & 0.67 \\
\hline SOC 56: other agricultural \& related occupations & $1133 / 1160$ & 1.0 & $51 / 51$ & $1.0[0.7-1.6]$ & $28 / 38$ & $0.7[0.4-1.3]$ & $23 / 13$ & $2.1[1.0-4.5]^{*}$ & 0.094 \\
\hline SOC 561: farm occupations, except managerial & 1159/1187 & 1.0 & $25 / 24$ & $1.2[0.6-2.1]$ & $14 / 18$ & $0.7[0.3-1.5]$ & $11 / 6$ & $3.2[1.0-10.1]^{*}$ & 0.060 \\
\hline SOC 5612: general farm workers & $1180 / 1205$ & 1.0 & $4 / 6$ & $0.9[0.3-2.9]$ & $1 / 4$ & $0.2[0.0-1.3 E+09]$ & $3 / 2$ & $3.3[0.4-27.8]$ & 0.36 \\
\hline SOC 5613: field crop \& vegetable farm workers & $1176 / 1202$ & 1.0 & $8 / 9$ & $1.0[0.3-3.0]$ & $4 / 6$ & $0.7[0.1-3.3]$ & $4 / 3$ & $1.7[0.2-11.2]$ & 0.69 \\
\hline SOC 562: related agricultural occupations & $1158 / 1183$ & 1.0 & $26 / 28$ & $0.9[0.5-1.6]$ & $14 / 21$ & $0.7[0.3-1.5]$ & $12 / 7$ & $1.6[0.5-5.0]$ & 0.61 \\
\hline SOC 61: mechanics and repairers & $1065 / 1081$ & 1.0 & $119 / 130$ & $0.8[0.6-1.1]$ & $36 / 25$ & $1.2[0.7-2.2]$ & $83 / 105$ & $0.7[0.5-1.0]^{*}$ & 0.038 \\
\hline SOC 644: painters, paperhangers, and plasterers & 1166/1193 & 1.0 & $18 / 18$ & $1.0[0.5-2.1]$ & $9 / 6$ & $1.0[0.3-3.3]$ & 9/12 & $1.1[0.4-3.1]$ & 0.88 \\
\hline SOC 681-682: precision metal workers & $1137 / 1177$ & 1.0 & $47 / 34$ & $1.2[0.8-1.9]$ & $14 / 10$ & $1.4[0.6-3.4]$ & $33 / 24$ & $1.2[0.7-2.0]$ & 0.48 \\
\hline SOC 7643: printing machine operators \& tenders & $1176 / 1205$ & 1.0 & $8 / 6$ & $1.3[0.4-3.9]$ & $6 / 3$ & $2.1[0.4-10.4]$ & $2 / 3$ & $0.5[0.0-5.2]$ & 0.71 \\
\hline $\begin{array}{l}\text { SOC 7658: laundering and dry-cleaning machine } \\
\text { operators and tenders }\end{array}$ & $1177 / 1202$ & 1.0 & $7 / 9$ & $0.8[0.3-2.3]$ & $4 / 6$ & $0.6[0.1-2.4]$ & $3 / 3$ & $1.2[0.2-8.7]$ & 0.997 \\
\hline SOC 772: assemblers & 1043/1085 & 1.0 & $141 / 126$ & $1.1[0.9-1.5]$ & $67 / 55$ & $1.2[0.8-1.8]$ & $74 / 71$ & $1.1[0.7-1.6]$ & 0.61 \\
\hline SOC 821: motor vehicle operators & $1030 / 1085$ & 1.0 & $154 / 126$ & $1.2[0.9-1.6]$ & $62 / 49$ & $1.3[0.9-2.1]$ & $92 / 77$ & $1.1[0.8-1.6]$ & 0.56 \\
\hline SOC 8243: sailors and deckhands & $1177 / 1202$ & 1.0 & $7 / 9$ & $1.2[0.4-3.4]$ & $3 / 2$ & $1.7[0.2-17.0]$ & $4 / 7$ & $1.0[0.3-3.5]$ & 0.93 \\
\hline \multicolumn{10}{|l|}{ INDUSTRY: } \\
\hline SIC 01: agricultural production, crops & 1161/1191 & 1.0 & $23 / 20$ & $1.4[0.7-2.8]$ & $13 / 15$ & $0.9[0.4-2.3]$ & $10 / 5$ & $3.3[1.0-11.5]$ & 0.051 \\
\hline SIC 02: agricultural production, livestock & $1180 / 1204$ & 1.0 & $4 / 7$ & $0.4[0.1-1.7]$ & $3 / 5$ & $0.5[0.1-2.6]$ & $1 / 2$ & $0.3[0.0-1.5 \mathrm{E}+09]$ & 0.61 \\
\hline SIC 29: petroleum and coal productscpe & $1177 / 1207$ & 1.0 & $7 / 4$ & $1.6[0.4-6.8]$ & $2 / 1$ & $2.0[0.0-1.4 \mathrm{E}+11]$ & $5 / 3$ & $1.4[0.3-6.1]$ & 0.59 \\
\hline SIC 3312: blast furnaces and steel mills & $1142 / 1164$ & 1.0 & $42 / 47$ & $0.8[0.5-1.3]$ & $20 / 17$ & $1.5[0.8-2.9]$ & $22 / 30$ & $0.5[0.3-1.0]^{*}$ & 0.061 \\
\hline SIC 7216: dry-cleaning plants, except rug & $1169 / 1198$ & 1.0 & $15 / 13$ & $2.0[0.9-4.4]$ & $11 / 10$ & $1.8[0.6-5.4]$ & $4 / 3$ & $2.5[0.4-14.4]$ & 0.093 \\
\hline
\end{tabular}

a Adjusted for sex, age at reference date, race, study center, education level, history of hypertension, smoking status, BMI ( 5 years prior to interview) and family history of cancer. ${ }^{*}$-value $<0.05$. $P$-trends $\leq 0.05$ for duration (Never, $<5$ years, $5+$ years) of employment are bolded. Results presented if a job was held by $\geq 10$ participants. Occupational results for architects, aircraft mechanics, railway workers, fisherman, sailors, seafarers, petroleum workers, and structural and sheet metal workers not shown due to small numbers. Industry results for blast furnace, coke oven, iron and steel, paper, and petroleum refining not shown due to small numbers. 
Table 3 Risk of renal cell carcinoma for a posteriori high- and low-risk occupations

\begin{tabular}{|c|c|c|c|c|c|c|c|c|c|}
\hline \multirow[t]{2}{*}{ Job Code and Description } & \multirow{2}{*}{\multicolumn{2}{|c|}{$\frac{\text { NEVER }}{\text { Case/Control }}$}} & \multicolumn{2}{|c|}{ EVER } & \multicolumn{2}{|c|}{$<5$ YEARS } & \multicolumn{2}{|c|}{ 5+ YEARS } & \multirow[t]{2}{*}{$P$-trend } \\
\hline & & & Case/Control & $\mathrm{OR}^{\mathrm{a}}[95 \% \mathrm{Cl}]$ & Case/Control & $\mathrm{OR}^{\mathrm{a}}[95 \% \mathrm{Cl}]$ & Case/Control & $\mathrm{OR}^{\mathrm{a}}[95 \% \mathrm{Cl}]$ & \\
\hline $\begin{array}{l}\text { SOC 128: administrators, education \& } \\
\text { related fields }\end{array}$ & $1179 / 1192$ & 1.0 & $5 / 19$ & $0.3[0.1-0.8]^{*}$ & $1 / 3$ & $0.20[0.0-1.9 \mathrm{E}+09]$ & $4 / 16$ & $0.3[0.1-0.9]^{*}$ & 0.037 \\
\hline SOC 171: computer scientists ${ }^{b}$ & $1180 / 1195$ & 1.0 & $4 / 16$ & $0.2[0.1-0.7]^{*}$ & $1 / 7$ & $0.1[0.0-0.7]^{*}$ & $3 / 9$ & $0.3[0.1-1.1]$ & 0.054 \\
\hline SOC 2033: recreation workers & $1175 / 1206$ & 1.0 & $9 / 5$ & $4.8[1.7-13.9]^{*}$ & $7 / 5$ & -——-——- & $2 / 0$ & -—-ー-一ー & \\
\hline SOC 25: librarians, archivists, and curators & $1182 / 1203$ & 1.0 & $2 / 8$ & $0.2[0.1-1.0]^{*}$ & $1 / 3$ & $0.2[0.0-1.3]$ & $1 / 5$ & $0.3[0.0-1.4]$ & 0.096 \\
\hline SOC 26: physicians and dentists & $1179 / 1198$ & 1.0 & $5 / 13$ & $0.4[0.2-0.8]^{*}$ & $1 / 2$ & $0.5[0.0-6.8]$ & $4 / 11$ & $0.4[0.1-1.1]$ & 0.067 \\
\hline SOC 36: health technologists and technicians & $1135 / 1175$ & 1.0 & $49 / 36$ & $1.7[1.1-2.6]^{*}$ & $19 / 14$ & $1.7[0.8-3.8]$ & $30 / 22$ & $1.6[1.0-2.7]$ & 0.043 \\
\hline SOC 397: programmers & $1177 / 1195$ & 1.0 & $7 / 16$ & $0.3[0.1-0.9]^{*}$ & $1 / 2$ & $0.3[0.0-1.0 \mathrm{E}+09]$ & $6 / 14$ & $0.3[0.1-1.0]^{*}$ & 0.042 \\
\hline SOC 403: supervisors: sales occupations, retail & $1126 / 1133$ & 1.0 & $58 / 78$ & $0.7[0.5-1.0]^{*}$ & $15 / 29$ & $0.4[0.2-0.7]^{*}$ & $43 / 49$ & $0.9[0.5-1.4]$ & 0.36 \\
\hline $\begin{array}{l}\text { SOC 46-47: administrative support } \\
\text { occupations, including clerical }\end{array}$ & $743 / 685$ & 1.0 & $441 / 526$ & $0.8[0.6-0.9]^{*}$ & 128/161 & $0.8[0.6-1.1]$ & $313 / 365$ & $0.7[0.6-0.9]^{*}$ & 0.0086 \\
\hline SOC 462: secretaries, stenographers and typists & $1064 / 1062$ & 1.0 & $120 / 149$ & $0.7[0.5-1.0]^{*}$ & $27 / 49$ & $0.5[0.3-0.8]^{*}$ & $93 / 100$ & $0.8[0.6-1.2]$ & 0.31 \\
\hline SOC 4696: file clerks & 1174/1181 & 1.0 & $10 / 30$ & $0.4[0.2-0.7]^{*}$ & $7 / 22$ & $0.4[0.2-0.7]^{*}$ & $3 / 8$ & $0.4[0.1-1.4]$ & 0.069 \\
\hline SOC 4715: billing clerks & $1177 / 1193$ & 1.0 & $7 / 18$ & $0.4[0.2-1.0]^{*}$ & $5 / 12$ & $0.4[0.1-1.4]$ & $2 / 6$ & $0.3[0.1-0.8]^{*}$ & 0.019 \\
\hline $\begin{array}{l}\text { SOC 474: mail \& message distributing } \\
\text { occupations }{ }^{c}\end{array}$ & $1141 / 1146$ & 1.0 & $43 / 65$ & $0.7[0.4-1.1]$ & $22 / 27$ & $0.8[0.4-1.6]$ & $21 / 38$ & $0.5[0.3-1.0]$ & 0.050 \\
\hline $\begin{array}{l}\text { SOC 475: material recording, scheduling \& } \\
\text { distributing clerks }\end{array}$ & 1091/1094 & 1.0 & $93 / 117$ & $0.7[0.5-0.9]^{*}$ & $49 / 64$ & $0.7[0.5-1.1]$ & $44 / 53$ & $0.6[0.4-1.0]$ & 0.033 \\
\hline $\begin{array}{l}\text { SOC 5216: food counter, fountain \& related } \\
\text { occupations }\end{array}$ & $1165 / 1178$ & 1.0 & $19 / 33$ & $0.5[0.3-1.0]^{*}$ & $14 / 28$ & $0.5[0.3-1.0]^{*}$ & $5 / 5$ & $0.6[0.1-3.0]$ & 0.18 \\
\hline SOC 525-526: personal service occupations & $1117 / 1104$ & 1.0 & $67 / 107$ & $0.7[0.5-0.9]^{*}$ & $37 / 72$ & $0.6[0.4-0.9]^{*}$ & $30 / 35$ & $0.8[0.5-1.4]$ & 0.18 \\
\hline SOC 5263: welfare service aides & 1178/1194 & 1.0 & $6 / 17$ & $0.4[0.2-1.0]^{*}$ & $3 / 12$ & $0.3[0.1-1.1]$ & $3 / 5$ & $0.6[0.2-2.1]$ & 0.20 \\
\hline $\begin{array}{l}\text { SOC 615: electrical \& electronic equipment } \\
\text { repairers }{ }^{d}\end{array}$ & $1157 / 1162$ & 1.0 & $27 / 49$ & $0.5[0.3-0.8]^{*}$ & $7 / 13$ & $0.6[0.2-1.6]$ & $20 / 36$ & $0.5[0.3-0.8]^{*}$ & 0.0065 \\
\hline SOC 7657: pressing machine operators & $1177 / 1207$ & 1.0 & $7 / 4$ & $4.7[1.3-17.4]^{*}$ & $5 / 3$ & $5.1[0.8-30.5]$ & $2 / 1$ & $4.1[1.0-17.8]$ & 0.044 \\
\hline SOC 8725: machine feeders and off-bearers & 1158/1197 & 1.0 & $26 / 14$ & $2.2[1.1-4.3]^{*}$ & $16 / 10$ & 1.9 [0.8-4.1] & $10 / 4$ & $3.2[1.1-9.3]^{*}$ & 0.024 \\
\hline
\end{tabular}

${ }^{a}$ Adjusted for sex, age at reference date, race, study center, education level, history of hypertension, smoking status, BMI (5 years prior to interview) and family history of cancer. Similar patterns of association were observed for: ${ }^{b}$ SOC 171 and $1719 ;{ }^{c}$ SOC 474 and 4743; ${ }^{d}$ SOC 615, 6151, and 6158* * -value $<0.05$. P-trends $<0.05$ for duration (Never, $<5$ years, $5+$ years) of employment are bolded. Results presented for $a$ posteriori high- and low-risk occupations only if we observed (1) a significant association with ever employment or (2) both a significant association with duration of employment and $P$-value for ever employment of 0.10 or

lower. Results presented if a job was held by $>10$ participants. 
Table 4 Risk of renal cell carcinoma for a posteriori high- and low-risk industries

\begin{tabular}{|c|c|c|c|c|c|c|c|c|c|}
\hline \multirow[t]{2}{*}{ Job Code and Description } & \multirow{2}{*}{\multicolumn{2}{|c|}{$\frac{\text { NEVER }}{\text { Case/Control }}$}} & \multicolumn{2}{|c|}{ EVER } & \multicolumn{2}{|c|}{$<5$ YEARS } & \multicolumn{2}{|c|}{ 5+ YEARS } & \multirow[t]{2}{*}{$P$-trend } \\
\hline & & & Case/Control & $\mathrm{OR}^{\mathrm{a}}[95 \% \mathrm{Cl}]$ & Case/Control & $\mathrm{OR}^{\mathrm{a}}[95 \% \mathrm{Cl}]$ & Case/Control & $\mathrm{OR}^{\mathrm{a}}[95 \% \mathrm{Cl}]$ & \\
\hline SIC 1611: highway and street construction & $1168 / 1205$ & 1.0 & $16 / 6$ & $3.1[1.0-9.4]^{*}$ & $8 / 4$ & $1.9[0.6-6.4]$ & $8 / 2$ & $7.4[0.7-75.7]$ & 0.069 \\
\hline SIC 26: paper and allied products & $1162 / 1196$ & 1.0 & $22 / 15$ & $1.8[0.8-4.0]$ & $12 / 11$ & $1.3[0.5-3.6]$ & $10 / 4$ & $3.3[1.0-10.9]^{*}$ & 0.046 \\
\hline SIC 2711: newspapers & $1164 / 1176$ & 1.0 & $20 / 35$ & $0.5[0.3-0.9]^{*}$ & $14 / 23$ & $0.6[0.3-1.2]$ & $6 / 12$ & $0.3[0.1-1.0]^{*}$ & 0.023 \\
\hline SIC 4941: water supply & $1176 / 1209$ & 1.0 & $8 / 2$ & $4.3[1.1-16.2]^{*}$ & $3 / 0$ & -ー-ーー-ー & $5 / 2$ & -ー-ー-ー- & \\
\hline $\begin{array}{l}\text { SIC 507: hardware, plumbing heating } \\
\text { equipment supplies }\end{array}$ & $1173 / 1210$ & 1.0 & $11 / 1$ & $6.9[1.4-33.6]^{*}$ & $7 / 0$ & -ーーー——- & $4 / 1$ & -——-—— & \\
\hline SIC 5461: retail bakeries & $1170 / 1203$ & 1.0 & $14 / 8$ & $2.1[0.9-4.8]$ & $7 / 6$ & $1.6[0.5-4.7]$ & $7 / 2$ & $3.7[1.0-14.2]$ & 0.046 \\
\hline SIC 62: security, commodity brokers \& services & $1170 / 1203$ & 1.0 & $14 / 8$ & $3.2[1.2-8.7]^{*}$ & $5 / 5$ & $2.0[0.5-9.2]$ & $9 / 3$ & $4.6[1.3-16.2]^{*}$ & 0.015 \\
\hline SIC 73: business services ${ }^{b}$ & $1049 / 1033$ & 1.0 & $135 / 178$ & $0.7[0.5-0.9]^{*}$ & $71 / 91$ & $0.7[0.5-1.1]$ & $64 / 87$ & $0.6[0.4-0.8]^{*}$ & 0.0012 \\
\hline $\begin{array}{l}\text { SIC 737: computer programming, data processing, } \\
\text { other repair }\end{array}$ & $1165 / 1184$ & 1.0 & $19 / 27$ & $0.6[0.3-1.0]$ & $8 / 7$ & $1.0[0.4-2.6]$ & $11 / 20$ & $0.5[0.2-1.0]^{*}$ & 0.038 \\
\hline SIC 738: miscellaneous business services & $1132 / 1147$ & 1.0 & $52 / 64$ & $0.7[0.5-1.1]$ & $27 / 42$ & $0.6[0.4-1.0]^{*}$ & $25 / 22$ & $0.9[0.5-1.7]$ & 0.56 \\
\hline SIC 8062: general medical and surgical hospitals & $1177 / 1194$ & 1.0 & $7 / 17$ & $0.4[0.1-0.9]^{*}$ & $0 / 8$ & ーーーー一ー & $7 / 9$ & ー-ー-ー一 & \\
\hline SIC 8069: specialty hospitals, except psychiatric & $1181 / 1196$ & 1.0 & $3 / 15$ & $0.2[0.0-1.0]^{*}$ & $3 / 8$ & -————— & $0 / 7$ & -——-—— & \\
\hline SIC 8082: home health care services & $1171 / 1202$ & 1.0 & $13 / 9$ & $2.8[1.1-7.2]^{*}$ & $8 / 4$ & $5.2[1.5-18.2]^{*}$ & $5 / 5$ & $1.4[0.3-6.7]$ & 0.41 \\
\hline SIC 82: educational services ${ }^{c}$ & $1020 / 992$ & 1.0 & $164 / 219$ & $0.7[0.6-0.9]^{*}$ & $77 / 91$ & $0.8[0.6-1.1]$ & $87 / 128$ & $0.6[0.5-0.8]^{*}$ & 0.0018 \\
\hline SIC 8361: residential care & $1171 / 1176$ & 1.0 & $13 / 35$ & $0.4[0.2-0.9]^{*}$ & $5 / 17$ & $0.4[0.1-1.0]$ & $8 / 18$ & $0.5[0.2-1.4]$ & 0.13 \\
\hline SIC 86: membership organizations & $1151 / 1158$ & 1.0 & $33 / 53$ & $0.6[0.4-1.0]$ & $16 / 20$ & $0.8[0.4-1.7]$ & $17 / 33$ & $0.5[0.3-1.0]$ & 0.051 \\
\hline $\begin{array}{l}\text { SIC 91: executive, legislative \& general } \\
\text { government }{ }^{d}\end{array}$ & $1173 / 1183$ & 1.0 & $11 / 28$ & $0.4[0.2-0.8]^{*}$ & $7 / 8$ & $0.8[0.2-2.9]$ & $4 / 20$ & $0.2[0.1-0.7]^{*}$ & 0.011 \\
\hline SIC 9221: police protection ${ }^{\mathrm{e}}$ & $1158 / 1195$ & 1.0 & $26 / 16$ & $2.2[1.0-4.8]^{*}$ & $3 / 4$ & $1.3[0.2-7.4]$ & $23 / 12$ & $2.4[1.0-5.7]^{*}$ & 0.045 \\
\hline SIC 97: national security \& international affairs ${ }^{f}$ & $962 / 966$ & 1.0 & $222 / 245$ & $0.8[0.6-1.0]^{*}$ & $122 / 135$ & $0.7[0.6-1.0]^{*}$ & $100 / 110$ & $0.9[0.6-1.1]$ & 0.29 \\
\hline
\end{tabular}

${ }^{a}$ Adjusted for sex, age at reference date, race, study center, education level, history of hypertension, smoking status, BMI (5 years prior to interview) and family history of cancer. Similar patterns of association were observed for: ${ }^{\mathrm{b}} \mathrm{SIC} 73,734$ and 7349 ; $^{\mathrm{c}} \mathrm{SIC} 82$ and 8221 ; $^{\mathrm{d}} \mathrm{SIC} 91$ and 9199 ; ${ }^{\mathrm{e}} \mathrm{SIC} 92,922,9221 ;{ }^{\mathrm{f}}$ SIC 97 and $9711 .{ }^{*} P$-value $<0.05$. $P$-trends $<0.05$ for duration (Never, $<5$ years, $5+$ years) of employment are bolded. Results presented for $a$ posteriori high- and low-risk industries only if we observed (1) a significant association with ever employment or (2) both a significant association with duration of employment and $P$-value for ever employment of 0.10 or lower. Results presented if a job was held by $\geq 10$ participants. 
paper and allied products (SIC 26: OR $=3.3[95 \% \mathrm{CI}=$ 1.0-10.9], $P$ for trend=0.046) industry. Notable increased associations without significant duration trends were seen in highway and street construction (SIC 1611: $\mathrm{OR}=3.1 \quad$ [95\% CI=1.0-9.4]), water supply (SIC 4941: $\mathrm{OR}=4.3 \quad[95 \% \mathrm{CI}=1.1-16.2]$ ), hardware/ plumbing/heating equipment supply (SIC 507: OR =6.9 [95\% CI =1.4-33.6]), and home health care services (SIC 8082: $\mathrm{OR}=2.8[95 \% \mathrm{CI}=1.1-7.2])$ industries. Industries with sex-specific associations that were statistically significant for ever employment and had significant trends with duration of employment, other than those mentioned above, were the electronic computers industry (SIC 3571: $\mathrm{OR}=0.3 \quad[95 \% \mathrm{CI}=0.1-1.0], P$ for trend $=$ 0.017 ) for men, and the electrical and electronics equipment industry (SIC 36: OR $=2.3$ [95\% CI = 1.0-5.2], $P$ for trend $=0.010)$ and motor vehicle parts and accessories (SIC 3714: $\mathrm{OR}=2.1 \quad[95 \% \mathrm{CI}=1.2-3.8], P$ for trend $=$ 0.014 ) for women; race-specific analyses did not identify additional industries meeting these criteria (Additional file 1: Table S1). Inverse associations with RCC risk were observed for those employed in the government, education, computer programming, membership organizations or business service industries.

Significant associations between ccRCC and a priori and a posteriori occupations and industries from above are shown in Table 5. Most associations remained essentially unchanged when analyses were restricted to cases with clear cell histologic subtype. However, the OR increased for the agricultural crops industry (SOC 01: $\mathrm{OR}=3.0 \quad[95 \% \mathrm{CI}=1.0-8.9])$, and the trend with duration of employment became statistically significant $(P=0.0050)$. Similarly, for agricultural and related occupations, excluding farm managers and proprietors (SOC 56 ), the OR for employment of five years or longer increased to 3.1 (95\% CI=1.4-6.8), with a now significant trend with duration of employment $(P=0.0096)$; the association was particularly strong for nonmanagerial farm occupations (SOC 561: OR $=5.9[95 \%$ $\mathrm{CI}=1.8-19.0], \quad P$ for trend $=0.0020)$. Associations also strengthened somewhat for employment in the drycleaning plant (SOC 7216: $\mathrm{OR}=3.0$ [95\% CI =1.2-7.4], $P$ for trend $=0.031$ ) and the private household industries (SIC 88: OR $=2.4$ [95\% CI $=1.3-4.4], P$ for trend $=$ 0.018 ), and for private household cleaners and servants (SOC 507: $\mathrm{OR}=3.5 \quad[95 \% \quad \mathrm{CI}=1.2-10.2], \quad P \quad$ for trend $=0.029$ ).

\section{Discussion}

Several occupations and industries were associated with significantly elevated RCC risk in this study. Of particular interest are the findings for employment in the agricultural and dry-cleaning industries, both of which have been previously associated with RCC. These associations became stronger when the analysis was restricted to patients with ccRCC, as was the case for private household cleaners and servants.

Increased RCC risk has been reported for agricultural/ farming jobs in many [8-12,25-27], but not all [28,29], epidemiologic studies. Elevated risk of RCC was observed for agricultural and animal husbandry workers, dairy and general farmers, field crop and vegetable workers, and farm machinery operators in a large Eastern European multi-center case-control study. The majority of these jobs were also observed to have higher risk associated with longer duration of employment [8]. Among men, a nearly two-fold increase in RCC risk was shown for general farm $[9,12]$ and horticultural [9] workers in two separate population-based case-control studies in Iowa and Canada. Additionally, elevated renal cancer mortality (standardized mortality ratio $(\mathrm{SMR})=$ 2.12) was reported in a cohort study of farmers in Italy [25], while a significant excess in kidney cancer death (proportionate mortality rate $=1.10$ ) was found among Caucasian farmers across 23 U.S. states [26]. Recent updates to the National Cancer Institute's Agricultural Health Study (AHS) also reported a significantly elevated kidney cancer mortality risk for farmers (relative SMR = 1.62) [27]. However, important evidence to the contrary also showed a significant $18 \%$ to $39 \%$ reduction in kidney/renal pelvis cancer incidence among AHS farmers and their spouses [28]. Furthermore, an earlier review of cancer patterns among farmers based on reports from 13 studies of varying designs across industrialized countries found a significant $8 \%$ reduction (meta-relative risk $=0.92,95 \% \mathrm{CI}=0.86-0.98$ ) in kidney cancer risk [29]. While pesticides [8,30-32] have been postulated as the exposure responsible for the elevated RCC risk observed among agricultural workers in some studies, these workers may also be exposed to a variety of other potentially carcinogenic substances, including chlorinated solvents, metals, fertilizers, engine exhaust, animal viruses, and microbes.

Although we did not observe significant associations with RCC risk for the other a priori occupations or industries, there was a non-significant doubling of risk in the dry-cleaning industry, an association that strengthened when restricted to patients with clear cell RCC. Previous studies have associated dry-cleaning industry workers with RCC [15,33]; none investigated associations with ccRCC. We also observed non-significant elevations in RCC risk for individuals in the cleaning and janitorial services, paper and allied products, electronics, motor vehicle, and auto repair industries, and with ccRCC for private household cleaners and servants. Researchers have speculated that the increased RCC risk observed for these occupations may be related to solvent exposures [34-37]. In particular, tetrachloroethylene 
Table 5 Risk of clear cell renal cell carcinoma for select occupations and industries

\begin{tabular}{|c|c|c|c|c|c|c|c|c|c|}
\hline \multirow[t]{2}{*}{ Job Code \& Description } & \multirow{2}{*}{\multicolumn{2}{|c|}{$\frac{\text { NEVER }}{\text { Case/Control }}$}} & \multicolumn{2}{|c|}{ EVER } & \multicolumn{2}{|c|}{$<5$ YEARS } & \multicolumn{2}{|c|}{ 5+ YEARS } & \multirow[t]{2}{*}{$P$-trend } \\
\hline & & & Case/Control & $\mathrm{OR}^{\mathrm{a}}[95 \% \mathrm{Cl}]$ & Case/Control & $\mathrm{OR}^{\mathrm{a}}[95 \% \mathrm{Cl}]$ & Case/Control & $\mathrm{OR}^{\mathrm{a}}[95 \% \mathrm{Cl}]$ & \\
\hline \multicolumn{10}{|l|}{ OCCUPATION: } \\
\hline SOC 171: computer scientists & $688 / 1195$ & 1.0 & $2 / 16$ & $0.2[0.0-1.0]^{*}$ & $1 / 7$ & $0.2[0.0-1.2]$ & $1 / 9$ & $0.2[0.0-1.7 E+09]$ & 0.67 \\
\hline SOC 36: health technologists and technicians ${ }^{b}$ & $656 / 1175$ & 1.0 & $34 / 36$ & $2.0[1.2-3.2]^{*}$ & $13 / 14$ & $2.1[0.9-4.8]$ & $21 / 22$ & $1.9[1.0-3.7]^{*}$ & 0.030 \\
\hline SOC 4122: insurance sales occupations & $674 / 1195$ & 1.0 & $16 / 16$ & $2.2[1.0-4.8]$ & $5 / 9$ & $1.1[0.4-2.9]$ & $11 / 7$ & $3.4[1.1-10.1]^{*}$ & 0.029 \\
\hline $\begin{array}{l}\text { SOC 4242: sales representatives, commercial and } \\
\text { industrial equipment/supplies }\end{array}$ & $675 / 1199$ & 1.0 & $15 / 12$ & $2.4[1.1-5.6]^{*}$ & $3 / 5$ & $1.4[0.3-7.4]$ & $12 / 7$ & $3.0[1.1-8.2]^{*}$ & 0.028 \\
\hline $\begin{array}{l}\text { SOC 46-47: administrative support occupations, } \\
\text { including clerical }\end{array}$ & $429 / 685$ & 1.0 & $261 / 526$ & $0.8[0.6-0.9]^{*}$ & $81 / 161$ & $0.8[0.6-1.1]$ & $180 / 365$ & $0.7[0.6-0.9]^{*}$ & 0.012 \\
\hline SOC 4696: file clerks & $685 / 1181$ & 1.0 & $5 / 30$ & $0.3[0.2-0.8]^{*}$ & $4 / 22$ & $0.4[0.1-0.8]^{*}$ & $1 / 8$ & $0.3[0.1-1.4]$ & 0.059 \\
\hline $\begin{array}{l}\text { SOC 475: material recording, scheduling \& } \\
\text { distributing clerks }\end{array}$ & $639 / 1094$ & 1.0 & $51 / 117$ & $0.6[0.4-0.9]^{*}$ & $27 / 64$ & $0.6[0.4-1.0]$ & $24 / 53$ & $0.6[0.4-1.1]$ & 0.048 \\
\hline SOC 507: private household cleaners \& servants ${ }^{c}$ & $681 / 1201$ & 1.0 & $9 / 10$ & $3.5[1.2-10.2]^{*}$ & $5 / 8$ & $2.7[0.7-10.7]$ & $4 / 2$ & $6.7[1.0-47.1]$ & 0.029 \\
\hline SOC 56: other agricultural and related occupations & $655 / 1160$ & 1.0 & $35 / 51$ & $1.2[0.8-2.0]$ & $17 / 38$ & $0.7[0.3-1.5]$ & $18 / 13$ & $3.1[1.4-6.8]^{*}$ & 0.0096 \\
\hline SOC 561: farm occupations, except managerial & $671 / 1187$ & 1.0 & $19 / 24$ & $1.6[0.8-3.2]$ & $9 / 18$ & $0.8[0.3-1.9]$ & $10 / 6$ & $5.9[1.8-19.0]^{*}$ & 0.0020 \\
\hline SOC 61: mechanics and repairers ${ }^{d}$ & $628 / 1081$ & 1.0 & $62 / 130$ & $0.7[0.5-1.0]$ & $20 / 25$ & $1.1[0.5-2.2]$ & $42 / 105$ & $0.6[0.4-0.9]^{*}$ & 0.024 \\
\hline SOC 687: precision food production occupations & $682 / 1207$ & 1.0 & $8 / 4$ & $3.5[1.1-10.8]^{*}$ & $3 / 1$ & $11.1[0.0-3.5 E+10]$ & $5 / 3$ & $2.3[0.6-9.1]$ & 0.14 \\
\hline \multicolumn{10}{|l|}{ INDUSTRY: } \\
\hline SIC 01: agricultural production, crops & $675 / 1191$ & 1.0 & $15 / 20$ & $3.0[1.0-8.9]^{*}$ & $6 / 15$ & $0.6[0.2-2.1]$ & $9 / 5$ & $6.3[1.7-23.3]^{*}$ & 0.0050 \\
\hline SIC 5461: retail bakeries & $680 / 1203$ & 1.0 & $10 / 8$ & $2.4[1.1-5.5]^{*}$ & $5 / 6$ & $1.8[0.7-5.0]$ & $5 / 2$ & $4.5[1.0-20.6]$ & 0.033 \\
\hline SIC 62: security, commodity brokers and services ${ }^{e}$ & $680 / 1203$ & 1.0 & $10 / 8$ & $3.5[1.2-10.0]^{*}$ & $3 / 5$ & $2.1[0.4-10.5]$ & $7 / 3$ & $5.2[1.4-19.5]^{*}$ & 0.013 \\
\hline SIC 7216: dry-cleaning plants, except rug & $677 / 1198$ & 1.0 & $13 / 13$ & $3.0[1.2-7.4]^{*}$ & $10 / 10$ & $2.9[0.9-9.1]$ & $3 / 3$ & $3.7[0.6-25.4]$ & 0.031 \\
\hline SIC 73: business services ${ }^{f}$ & $614 / 1033$ & 1.0 & $76 / 178$ & $0.6[0.5-0.9]^{*}$ & $37 / 91$ & $0.7[0.4-1.1]$ & $39 / 87$ & $0.6[0.4-0.9]^{*}$ & 0.0046 \\
\hline $\begin{array}{l}\text { SIC 737: computer programming, data processing, } \\
\text { other repair }\end{array}$ & $679 / 1184$ & 1.0 & $11 / 27$ & $0.5[0.3-1.0]$ & $5 / 7$ & $1.0[0.3-2.9]$ & $6 / 20$ & $0.4[0.2-0.9]^{*}$ & 0.024 \\
\hline SIC 8069: specialty hospitals, except psychiatric & $689 / 1196$ & 1.0 & $1 / 15$ & $0.1[0.0-0.5]^{*}$ & $1 / 8$ & -——-ー—— & $0 / 7$ & -—-—-—- & \\
\hline SIC 8082: home health care services & $682 / 1202$ & 1.0 & $8 / 9$ & $3.2[1.1-9.6]^{*}$ & $5 / 4$ & $6.7[1.6-28.4]^{*}$ & $3 / 5$ & $1.4[0.2-8.6]$ & 0.45 \\
\hline$\overline{S I C}$ 82: educational services ${ }^{9}$ & $596 / 992$ & 1.0 & $94 / 219$ & $0.7[0.5-0.9]^{*}$ & $40 / 91$ & $0.7[0.4-1.0]^{*}$ & $54 / 128$ & $0.7[0.5-0.9]^{*}$ & 0.014 \\
\hline SIC 8361: residential care & $684 / 1176$ & 1.0 & $6 / 35$ & $0.4[0.2-0.9]^{*}$ & $4 / 17$ & $0.6[0.2-1.8]$ & $2 / 18$ & $0.2[0.0-1.2]$ & 0.055 \\
\hline SIC 88: private households & $670 / 1176$ & 1.0 & $20 / 35$ & $2.4[1.3-4.4]^{*}$ & $11 / 21$ & $2.1[1.0-4.5]$ & $9 / 14$ & $2.9[1.1-7.7]^{*}$ & 0.018 \\
\hline SIC 919: general government, nec & $686 / 1188$ & 1.0 & $4 / 23$ & $0.4[0.1-1.0]^{*}$ & $2 / 5$ & $1.9[0.4-10.0]$ & $2 / 18$ & $0.2[0.1-0.6]^{*}$ & 0.0047 \\
\hline$\overline{\text { SIC 9221: police protection }{ }^{\mathrm{h}}}$ & $673 / 1195$ & 1.0 & $17 / 16$ & $2.5[1.1-5.8]^{*}$ & $2 / 4$ & $1.7[0.2-16.0]$ & $15 / 12$ & $2.6[1.0-6.7]^{*}$ & 0.039 \\
\hline SIC 97: national security and international affairs ${ }^{i}$ & $569 / 966$ & 1.0 & $121 / 245$ & $0.7[0.6-1.0]^{*}$ & $63 / 135$ & $0.7[0.5-0.9]^{*}$ & $58 / 110$ & $0.8[0.6-1.2]$ & 0.35 \\
\hline
\end{tabular}

${ }^{a}$ Adjusted for sex, age at reference date, race, study center, education level, history of hypertension, smoking status, BMI (5 years prior to interview) and family history of cancer. Similar patterns of association were observed for: ${ }^{\mathrm{b}}$ SOC 36 and $369 ;{ }^{\mathrm{c}}$ SOC 50 and 507; ${ }^{\mathrm{d}}$ SOC 615,6151 , and $6158 ;{ }^{\mathrm{e}}$ SIC 62 and $621 ;{ }^{\mathrm{f}}$ SIC 73 and $734 ;{ }^{9}$ SIC 82 and $8221 ;{ }^{\mathrm{h}}$ SIC 922 and $9221 ;{ }^{\mathrm{i}}$ SIC 97 and $971 .{ }^{*} P$-value $<0.05$. P-trends $\leq 0.05$ for duration (Never, $<5$ years, $5+$ years) of employment are bolded. Results presented if a job was held by $\geq 10$ participants. Results presented for occupations and industries associated with RCC risk except for SOC 507 and SIC 88 . 
(PCE), the primary solvent used in dry cleaning and frequently handled by metal and petroleum workers, and trichloroethylene (TCE), a chlorinated solvent commonly used as a degreaser in metal and automotive repair industries, have been studied extensively [3,34-38]. Evidence from animal and human studies have shown that exposure to these solvents induces nephrotoxicity and nephrocarcinogenicity [3,36,38,39]; both solvents have been classified by the International Agency for Research on Cancer as "probably carcinogenic to humans" [40]. Some epidemiological studies have linked TCE exposure to somatic mutation of the von Hippel-Lindau $(V H L)$ tumor suppressor gene which is thought to result in the development of the majority of ccRCCs $[41,42]$. These results suggest the nonrandom affinity of mutagenic TCE metabolites for VHL may lead to ccRCC, although other epidemiological studies investigating the association between TCE exposure, VHL damage, and RCC have not replicated these findings $[43,44]$. We interpret with caution the finding of elevated RCC risk for some typically solvent-exposed jobs in our study, given that we also observed a significant reduction in risk with increasing duration of employment among mechanics/ repairers, an occupation that carries a high probability of solvent exposure.

Other occupations and industries that were significantly associated with elevated RCC and/or ccRCC risk in our study included police and public safety workers, and health care workers and technicians. Non-significant increased renal cancer risk was observed among policemen in three different European cohort studies [10,45,46] and a large RCC case-control study in New Zealand [47]. No association with RCC was observed among male health care workers in a Swedish cohort study [10] or in a population-based RCC case-control study in Denmark [13], although a significant increase for kidney and renal pelvis cancer mortality risk (mortality $\mathrm{OR}=1.7$ ) was reported for black participants in a U.S. study of female health care workers [48]. In our study, risk for health care workers and technicians did not vary by race. Given the small number of subjects employed in most of the occupational categories reported above, additional studies are needed to replicate and/or confirm results.

Significantly reduced RCC risk was observed for several white-collar occupations in our study. Although most RCC occupational studies have not reported significant inverse associations for white-collar jobs, Heck and colleagues recently observed a significant 30\%-40\% reduction in renal cancer risk among Central and Eastern European clerical workers, business workers, and social workers [8]. We know of no mechanism by which these types of jobs may protect against renal cancer risk, and we suspect that these findings could be attributable to a combination of chance and confounding by unknown factors. Additional studies that include white-collar occupations are needed to clarify these possible associations.

Strengths of our study include its population-based design of Caucasians and African Americans, inclusion of only histologically confirmed RCC cancers, and large sample size. Further, our study of RCC is to our knowledge the first to evaluate associations with occupation and industry separately by race, and restricted to cases of clear cell histology. The associations with ccRCC observed in our study for employment in the agricultural and dry-cleaning industries suggest that the biologic effects underlying exposures in these occupations may be particularly relevant to the pathogenesis of this disease subtype. Subtype-specific investigations in other studies are needed to confirm these findings.

Our study also has limitations. Given the large number of occupations and industries evaluated and issues stemming from multiple comparisons, it is likely that some of our findings arose due to chance, particularly for the a posteriori occupations and industries and for subgroup analyses. In addition, similar to many recent populationbased studies, the response rates among the control subjects were not optimal. However, the sample weights included adjustments for differential nonresponse rates among demographic categories that may reduce bias in the analyses due to the nonresponse. The weights may also be useful in generalizing results to the Detroit and Chicago areas given that our control sources were approximately representative of the general population. Compared to the 2000 U.S. Census the population coverage rate for Chicago and Detroit for 20-64 year olds by the DMV records in 2002 (for Chicago) and 2003 (for Detroit) was about 100\% for males and 96\% for females. For Wayne County alone the coverage was lower, about $93 \%$ for males and $87.1 \%$ for females. We found the population coverage in Wayne County to be higher among 45-64 year olds, about 97\% for males and $92 \%$ for females, which was the age range of most of the cases [49]. The sample sizes for many of the occupations and industries were small, leading to wide confidence intervals. While we had sufficient power to detect relatively small main effects, the power for stratified analysis by sex and race was limited. Finally, job titles are only surrogates for exposure. A specific job title may be associated with a wide range of different possible exposures; grouping subjects who may be highly exposed with those potentially unexposed mitigates the strength of the association.

\section{Conclusions}

In summary, our findings from this large populationbased case-control study of Caucasians and African Americans offer support for associations with RCC in 
the agricultural and dry-cleaning industries, and suggest for the first time that these associations might be stronger for the ccRCC subtype. These findings, along with suggestive associations observed for other occupations and industries, offer new leads worthy of further epidemiologic investigation.

\section{Additional files}

Additional file 1: Table S1. Weighted analysis for renal cell carcinoma risk by industry, by sex.

Additional file 2: Table S2. Occupation and risk of renal cell carcinoma, by sex.

\section{Abbreviations}

AHS: Agricultural Health Study; BMI: Body mass index; ccRCC: Clear cell renal cell carcinoma; Cl: Confidence interval; DMV: Department of Motor Vehicle; ICD-O: International Classification of Disease for Oncology; OR: Odds ratio; PCE: tetrachloroethylene; RCC: Renal cell carcinoma; SIC: Standard Industry Classification; SMR: Standardized mortality ratio; SOC: Standard Occupational Classification; TCE: Trichloroethylene; U.S.: United States; USA: United States of America; VHL: Von Hippel-Lindau.

\section{Competing interests}

The authors declare that they have no competing interests.

\section{Authors' contributions}

KS, FGD, JJR, NR and W-HC designed the study and collected the data. SK, JSC, SSM, SW, PAS, BIG and MPP analyzed the data. SK, JSC, and MPP drafted the manuscript. All authors read, gave comments, and approved the final version of the manuscript. SK had full access to all the data in the study and take responsibility for the integrity of the data and accuracy of the data analysis. All authors have read and approved the final manuscript.

\section{Acknowledgements}

This study was supported by the Intramural Research Program of the National Institutes of Health and the National Cancer Institute with contracts NO2-CP-10128 (Westat, Inc.), N02-CP-11004 (Wayne State University), and N02-CP-11161 (University of Illinois at Chicago).

\section{Author details}

'Division of Cancer Epidemiology and Genetics, National Cancer Institute, NIH, DHHS, 6120 Executive Boulevard, MSC 7242, Bethesda, MD 20892-7242, USA. ${ }^{2}$ Wayne State University, Karmanos Cancer Institute, 110 E. Warren, Detroit, MI 48201, USA. ${ }^{3}$ University of Illinois, 877 SPHPI M/C 923, 1603 W. Taylor Street, Chicago, IL 60612, USA. ${ }^{4}$ Formerly of Information Management Service Inc, 6110 Executive Blvd Suite \#310, Rockville, MD 20852, USA. ${ }^{5}$ Stewart Exposure Assessments, LLC, Arlington, VA, USA. ${ }^{6}$ Formerly of the Division of Cancer Epidemiology and Genetics, National Cancer Institute, NIH, DHHS, Bethesda, MD, USA.

Received: 6 March 2012 Accepted: 9 July 2012

Published: 8 August 2012

\section{References}

1. McLaughlin JK, Lipworth L, Tarone RE: Epidemiologic aspects of renal cell carcinoma. Semin Oncol 2006, 33:527-533.

2. Jemal A, Siegel R, Ward E, Hao Y, Xu J, Thun MJ: Cancer Statistics, 2009. CA Cancer J Clin 2009, 59:225-249.

3. Chow WH, Devesa SS: Contemporary epidemiology of renal cell cancer. Cancer J 2008, 14:288-301.

4. Weikert S, Ljungberg B: Contemporary epidemiology of renal cell carcinoma: perspectives of primary prevention. World J Urol 2010, 28:247-252.

5. Störkel S, Eble JN, Adlakha K, Amin M, Blute ML, Bostwick DG, Darson M, Delahunt B, Iczkowski K: Classification of renal cell carcinoma: Workgroup
No. 1. Union Internationale Contre le Cancer (UICC) and the American Joint Committee on Cancer (AJCC). Cancer 1997, 80:987-989.

6. Cheville JC, Lohse CM, Zincke H, Weaver AL, Blute ML: Comparisons of outcome and prognostic features among histologic subtypes of renal cell carcinoma. Am J Surg Pathol 2003, 27:612-624.

7. Liu H, Hemminki K, Sundquist J: Renal cell carcinoma as first and second primary cancer: etiological clues from the Swedish Family-Cancer Database. J Urol 2011, 185:2045-2049.

8. Heck JE, Charbotel B, Moore LE, Karami S, Zaridze DG, Matveev V, Janout V, Kollárová H, Foretova L, Bencko V, Szeszenia-Dabrowska N, Lissowska J, Mates D, Ferro G, Chow WH, Rothman N, Stewart P, Brennan P, Boffetta P: Occupation and renal cell cancer in Central and Eastern Europe. Occup Environ Med 2010, 67:47-53.

9. Parent ME, Hua Y, Siemiatycki J: Occupational risk factors for renal cell carcinoma in Montreal. Am J Ind Med 2000, 38:609-618.

10. McLaughlin JK, Malker HS, Stone BJ, Weiner JA, Malker BK, Ericsson JL, Blot WJ, Fraumeni JF Jr: Occupational risks for renal cancer in Sweden. Br J Ind Med 1987, 44:119-123.

11. Mattioli S, Truffelli D, Baldasseroni A, Risi A, Marchesini B, Giacomini C, Bacchini P, Violante FS, Buiatti E: Occupational risk factors for renal cell cancer: a case-control study in northern Italy. J Occup Environ Med 2002, 44:1028-1036.

12. Zhang Y, Cantor KP, Lynch CF, Zheng T: A population-based case-control study of occupation and renal cell carcinoma risk in lowa. J Occup Environ Med 2004, 46:235-240.

13. Mellemgaard A, Engholm G, McLaughlin JK, Olsen JH: Occupational risk factors for renal-cell carcinoma in Denmark. Scand J Work Environ Health 1994, 20:160-165.

14. Brownson RC: A case-control study of renal cell carcinoma in relation to occupation, smoking, and alcohol consumption. Arch Environ Health 1988, 43:238-241.

15. Asal NR, Geyer JR, Risser DR, Lee ET, Kadamani S, Cherng N: Risk factors in renal cell carcinoma. II. Medical history, occupation, multivariate analysis, and conclusions. Cancer Detect Prev 1988, 13:263-279.

16. Mandel JS, McLaughlin JK, Schlehofer B, Mellemgaard A, Helmert U, Lindblad P, McCredie M, Adami HO: International renal-cell cancer study. IV. Occupation. Int J Cancer 1995, 61:601-605.

17. Colt JS, Schwartz K, Graubard BI, Davis F, Ruterbusch J, DiGaeteno R, Purdue M, Rothman N, Wacholder S, Chow WH: Hypertension and risk of renal cell carcinoma among white and black Americans. Epidemiol 2011, 22:797-804

18. US Department of Commerce: Standard Occupational Classification Manual. Washington, D.C.: Office of Federal Statistical Policy and Standards; 1980.

19. US Government Printing Office: Standard Industrial Classification Manual. Executive Office of the President, Office of Management and Budget: Washington, D.C; 1987.

20. Li Y, Graubard BI, DiGaetano R: Weighting methods for population-based case-control studies with complex sampling. J Royal Stat Soc Series C (Applied Statistics) 2011, 60:165-185.

21. Korn EL, Graubard Bl: Analysis of Health Surveys. New York: John Wiley \& Sons; 1990.

22. Rust KF, Rao JN: Variance estimation for complex surveys using replication techniques. Stat Methods Med Res 1996, 5:283-310.

23. Tellez-Plaza M, Navas-Acien A, Crainiceanu CM, Guallar E: Cadmium exposure and hypertension in the 1999-2004 National Health and Nutition Examination Survey (NHANES). Environ Health Perspect 2008, 116:51-56.

24. Navas-Acien A, Guallar E, Silbergeld EK, Rothenberg SJ: Lead exposure and cardiovascular disease-a systematic review. Environ Health Perspect 2007, 115:472-482.

25. Faustini A, Forastiere F, Di Betta L, Magliola EM, Perucci CA: Cohort study of mortality among farmers and agricultural workers. Med Lav 1993, 84:31-41.

26. Blair A, Dosemeci M, Heineman EF: Cancer and other causes of death among male and female farmers from twenty-three states. Am J Ind Med 1993, 23:729-742.

27. Waggoner JK, Kullman GJ, Henneberger PK, Umbach DM, Blair A, Alavanja MC, Kamel F, Lynch CF, Knott C, London SJ, Hines CJ, Thomas KW, Sandler DP, Lubin JH, Beane Freeman LE, Hoppin JA: Mortality in the agricultural health study, 1993-2007. Am J Epidemio/ 2011, 173(1):71-83. 
28. Koutros S, Alavanja MC, Lubin JH, Sandler DP, Hoppin JA, Lynch CF, Knott C, Blair A, Freeman LE: An update of cancer incidence in the Agricultural Health Study. J Occup Enviorn Med 2010, 52:1098-1105.

29. Blair A, Zahm SH, Pearce NE, Heineman EF, Fraumeni JF Jr: Clues to cancer etiology from studies of farmers. Scand J Work Environ Health 1992, 18:209-215.

30. Schreinemachers DM: Cancer mortality in four northern wheat-producing states. Environ Health Perspect 2010, 108:873-881.

31. Settimi L, Comba P, Bosia S, Ciapini C, Desideri E, Fedi A, Perazzo PL, Axelson O: Cancer risk among male farmers: a multi-site case-control study. Int J Occup Med Environ Health 2001, 14:339-347.

32. Karami S, Boffetta P, Rothman N, Hung RJ, Stewart T, Zaridze D, Navritalova M, Mates D, Janout V, Kollarova H, Bencko V, Szeszenia-Dabrowska N, Holcatova I, Mukeria A, Gromiec J, Chanock SJ, Brennan P, Chow WH, Moore LE: Renal cell carcinoma, occupational pesticide exposure and modification by glutathione S-transferase polymorphisms. Carcinogenesis 2008, 29:1567-1571.

33. McCredie M, Stewart JH: Risk factors for kidney cancer in New South Wales, IV. Occupation. Br J Ind Med 1993, 50:349-354

34. Moore LE, Boffetta P, Karami S, Brennan P, Stewart PS, Hung R, Zaridze D, Matveev V, Janout V, Kollarova H, Bencko V, Navratilova M, SzeszeniaDabrowska N, Mates D, Gromiec J, Holcatova I, Merino M, Chanock S, Chow $\mathrm{WH}$, Rothman N: Occupational trichloroethylene exposure and renal carcinoma risk: evidence of genetic susceptibility by reductive metabolism gene variants. Cancer Res 2010, 70:6527-6536.

35. Charbotel B, Fevotte J, Hours M, Martin JL, Bergeret A: Case-control study on renal cell cancer and occupational exposure to trichloroethylene. Part II: Epidemiological aspects. Ann Occup Hyg 2006, 50:777-787.

36. Centers for Diseases Prevention and Control: Trichloroethylene.

37. Bakke B, Stewart PA, Waters MA: Uses of and exposure to trichloroethylene in U.S. industry: a systematic literature review. J Occup Environ Hyg 2007, 4:375-390.

38. United States Environmental Protection Agency: Sources, emission and exposure for Trichloroethylene (TCE) and related chemicals. http://www.epa. gov/ncea/pdfs/tce/TCEMarch2001.pdf.

39. Pesch B, Haerting J, Ranft U, Klimpel A, Oelschlagel B, Schill W: Occupational risk factors for renal cell carcinoma: agent-specific results from a case-control study in Germany, MURC Study Group. Multicenter urothelial and renal cancer study. Int J Epidemiol 2000, 29:1014-1024.

40. IARC: Dry cleaning, some chlorinated solvents and other industrial chemicals. IARC Monogr Eval Carcinog Risks Hum 1995, 63:33-477.

41. Brauch $H$, Weirich $G$, Hornauer MA, Störkel S, Wöhl T, Brüning T: Trichloroethylene exposure and specific somatic mutations in patients with renal cell carcinoma. J Natl Cancer Inst 1999, 91:854-861.

42. Brauch $H$, Weirich $G$, Klein $B$, Rabstein $S$, Bolt HM, Brüning $T$ : VHL mutations in renal cell cancer: does occupational exposure to trichloroethylene make a difference? Toxicol Lett 2004, 151:301-310.

43. Charbotel B, Gad S, Caïola D, Béroud C, Fevotte J, Bergeret A, Ferlicot S, Richard S: Trichloroethylene exposure and somatic mutations of the VHL gene in patients with Renal Cell Carcinoma. J Occup Med Toxicol 2007, 2:13.

44. Moore LE, Nickerson ML, Brennan P, Toro JR, Jaeger E, Rinsky J, Han SS, Zaridze D, Matveev V, Janout V, Kollarova H, Bencko V, Navratilova M, Szeszenia-Dabrowska N, Mates D, Schmidt LS, Lenz P, Karami S, Linehan WM, Merino M, Chanock S, Boffetta P, Chow WH, Waldman FM, Rothman N: Von Hippel-Lindau (VHL) Inactivation in Sporadic Clear Cell Renal Cancer: Associations with Germline VHL Polymorphisms and Etiologic Risk Factors. PLoS Genet 2011, 7:e1002312.

45. Guo J, Kauppinen T, Kyyrönen P, Heikkila P, Lindbohm ML, Pukkala E: Risk of esophageal, ovarian, testicular, kidney and bladder cancers and leukemia among Finnish workers exposed to diesel or gasoline engine exhaust. Int J Cancer 2004, 111:286-292.

46. Forastiere F, Perucci CA, Di Pietro A, Miceli M, Rpiti E, Bargagli A, Borgia P: Mortality among urban policemen in Rome. Am J Ind Med 1994 26:785-798.

47. Delahunt B, Bethwaite PB, Nacey JN: Occupational risk for renal cel carcinoma. A case-control study based on the New Zealand Cancer Registry. Br J Urol 1995, 75:578-582.

48. Petralia SA, Dosemeci M, Adams EE, Zahm SH: Cancer mortality among women employed in health care occupations in 24 U.S. states, 1984-1993. Am J Ind Med 1999, 36:159-165.
49. DiGaetano R, Graubard B, Rao S, Severynse J, Wacholder S: Sampling racially matched population controls for case-control studies: using DMV lists and oversampling minorities. In Federal Committee on Statistical Methodology Research Conference 2003, Concurrent Session IX-B. FCSM Statistical Policy Working Paper 37. http://www.fcsm.gov/reports/.

doi:10.1186/1471-2407-12-344

Cite this article as: Karami et al:: A case-control study of occupation/ industry and renal cell carcinoma risk. BMC Cancer 2012 12:344.

\section{Submit your next manuscript to BioMed Central and take full advantage of:}

- Convenient online submission

- Thorough peer review

- No space constraints or color figure charges

- Immediate publication on acceptance

- Inclusion in PubMed, CAS, Scopus and Google Scholar

- Research which is freely available for redistribution

Submit your manuscript at www.biomedcentral.com/submit
C) Biomed Central 